The authors point out that the KDOQI IPTH target is based on data from 1990s studies and that management of bone metabolism in CKD has evolved substantially since then. As a result, other markers of bone remodeling might be needed to monitor renal osteodystrophy in stage 5 CKD.

Original article Barreto FC et al. (2008) K/DOQI-

recommended intact PTH levels do not prevent low-turnover bone disease in hemodialysis patients. Kidney Int 73: 771-777

\section{Parathyroid hormone measurements are influenced by blood sample processing}

Measurements of circulating parathyroid hormone (PTH) can be highly variable depending upon the assay used, but the standardization of blood sample handling might help to reduce this variability. To investigate the effects of blood handling conditions on PTH measurements, Joly et al. used six automated second-generation sandwich assays to compare PTH levels in blood specimens processed in three different ways. These assays measure so-called 'intact' PTH - that is, the entire PTH peptide plus PTH fragments.

Blood samples were obtained from 34 patients with stage 5 chronic kidney disease who were on chronic hemodialysis. Serum, EDTA plasma and sodium citrate plasma aliquots were obtained from each sample. Each aliquot was then tested for 'intact' PTH by use of six assays and was stored at room temperature for $16-22 \mathrm{~h}$ before being tested again. The type of assay used and the type of aliquot being tested significantly influenced the variability of PTH measurements ( $P<0.001$ for both), whereas time of measurement did not. In one assay, $7(20.6 \%)$ patients were classified differently according to the KDOQI categories for 'intact' PTH when serum or citrate plasma was used.

The best type of blood sample to use in PTH assays is not clearly evident from the study. Nonetheless, the authors suggest that serum samples should be used because PTH is stable in serum, calcium can be measured in the same sample, and plain tubes can be filled at less than $50 \%$.

Original article Joly D et al. (2008) Variation in serum and plasma PTH levels in second-generation assays in hemodialysis patients: a cross-sectional study. Am J Kidney Dis 51: 987-995

\section{Preventing rejection in sensitized kidney transplant recipients with donor-specific antibodies}

The optimum desensitization protocol for kidney transplant recipients with donor-specific anti-human leukocyte antigen antibodies (DSAs) and a negative complement-dependent cytotoxicity T-cell cross-match is unknown. Akalin et al. examined the efficacy of plasmapheresis and intravenous immunoglobulin (IVlg) in 35 such individuals.

All patients received induction therapy with Thymoglobulin ${ }^{\circledR}$ (Genzyme Corporation, Cambridge, MA) and high-dose IVlg during surgery and for an additional 2 days following surgery. The strength of each patient's DSA reaction to purified human leukocyte antigens was determined before transplantation through analysis of mean fluorescence intensity of Luminex ${ }^{\circledR}$ flow beads (Luminex Corporation, Austin, TX).

Acute rejection was observed in six (66\%) of the nine individuals with strong DSA reactions. As a result, all subsequently treated patients with strong DSA reactions received peritransplantation plasmapheresis in addition to IVlg. Only $1(7 \%)$ of these 14 patients developed acute rejection. None of the 12 patients with weak or moderate DSA reactions, who received high-dose IVIg only, had an acute rejection episode. During follow-up (median 18 months), the majority $(77 \%)$ of patients experienced complete or partial loss of DSAs. Graft survival was $100 \%$ in the patients with weak or moderate DSA reactions, $78 \%$ in the patients with strong DSA reactions who did not receive plasmapheresis, and $86 \%$ in the patients with strong DSA reactions who did receive plasmapheresis.

These data suggest that Thymoglobulin ${ }^{\circledR}$ and high-dose IVIg is sufficient for the prevention of acute rejection in kidney transplant recipients with a negative complement-dependent cytotoxicity T-cell cross-match who have weak or moderate DSA reactions, whereas patients with strong DSA reactions should be desensitized with plasmapheresis in addition to undergoing treatment with Thymoglobulin ${ }^{\circledR}$ and high-dose IVIg.

Original article Akalin E et al. (2008) Addition of plasmapheresis decreases the incidence of acute antibodymediated rejection in sensitized patients with strong donorspecific antibodies. Clin J Am Soc Nephrol [doi:10.2215/

CJN.05321107] 